\title{
AVALIAÇÄO DA INFESTAÇĀO E DIVERSIDADE DE TEREDINIDAE (MOLLUSCA - BIVALVIA) AO LONGO DA COSTA DO ESTADO DO RIO DE JANEIRO, BRASIL
}

\author{
ANDREA DE O. R. JUNQUEIRA, SERGIO HENRIQUE G. DA SILVA \& MARIA JÚLIA M. \\ SILVA
}

Departarnento de Biologia Marinha, Instituto de Biologia, Universidade lederal do Rio de Janeiro, Bl. A, CCS, Itha do Fundão, 21910 R 10 de Janciro, RJ, Brasil

Evaluation of the infestation and diversity of Teredinidae (Mollusca - Bivalvia) along the coast of Rio de Janeiro State, Brazil - In order to evaluate the diversity and infestation level of Teredinidae wood-boring mollusc's, ten stations were chosen along the coast of Rio de Janeiro State. Using five pine-wood panels in each station, it was possible to distinguish five areas according to species dominance.

Ilha Grande Bay is characterized by the dominance of Lyrodus floridanus (Bartsch, 1922); Sepetiba Bay, by Bankia fimbriatula Moll \& Roch, 1931, Tijuca Lagoon by Teredo navalis Linnaeus, 1758: Guanabara Bay by Nototeredo knoxi (Bartsch, 1917) and Araruama Lagoon by Teredo furcifera Martens, 1894.

Specific composition and dominance of these species were due mainly to abiotic factors such as salinity whereas infestation level was associated not only to salinity but also to other factors such as the quantity and quality of fouling, the presence of larval dispersion focus and the wood supply in each area.

Key words: Teredinidae - wood boring molluscs - bays - lagoons

O grupo mais importante entre os responsáveis pelos processos de deterioração da madeira no mar é constituído pelos moluscos bivalves perfurantes da família Teredinidae. Além dos grandes prejuízos que causam às instalações portuárias e embarcações de madeira estes organismos possuem um papel importante nos ecossistemas marinhos por contribuírem para a reciclagem da madeira (Turner, 1971; Turner \& Johnson, 1971). A distribuição das espécies de Teredinidae assim como o grau de infestação variam de região para região em função dos fatores que os controlam. Entre estes destacamse a presença de madeira, temperatura e salinidade da água, incrustação biológica e poluição. O conhecimento da distribuição e biologia das espécies que ocorrem no Brasil é condição indispensável quando se deseja sustar ou regular sua atividade já que fornece os dados que permitem estabelecer a sua vulnerabilidade aos agentes destruidores assim como informações que permitem uma aplicação racional e econômica de tais agentes (Fernandes \& Costa, 1967).

Trabalho realizado com o auxílio do CNPq.

\section{MATFRIAL E MÉTODOS}

As localidades escolhidas foram as seguintes: Canal de Itajuru (na área da Lagoa de Araruama), Urca (na área da Baía da Guanabara), Joá (na área da Lagoa da Tijuca), Barra de Guaratiba, Mangaratiba e Itacuruçá (na área da Baía de Sepetiba) e Portogallo, Angra dos Reis, Piraquara e Tarituba (na área da Baía da Ilha Grande) (Fig. 1). Utilizamos, para a coleta dos organismos, coletores de pinho (Araucaria angustifolia), constituídos de 10 lâminas de $10 \mathrm{~cm} \mathrm{x}$ $10 \mathrm{~cm} \times 0,08 \mathrm{~cm}$, justapostas e prensadas entre duas peças de cerâmica. Em cada localidade instalamos cinco coletores que permaneceram 3 meses imersos exceto no Canal de Itajuru (quatro meses). Após este período os coletores foram desmontados e as espécies identificadas. Para cada localidade foi calculado o Indice de Diversidade de Shannon-Wiener, com o logaritmo na base 10 (Washington, 1984). As estações foram comparadas entre si através do Indice de Sanders (Sanders, 1960). 


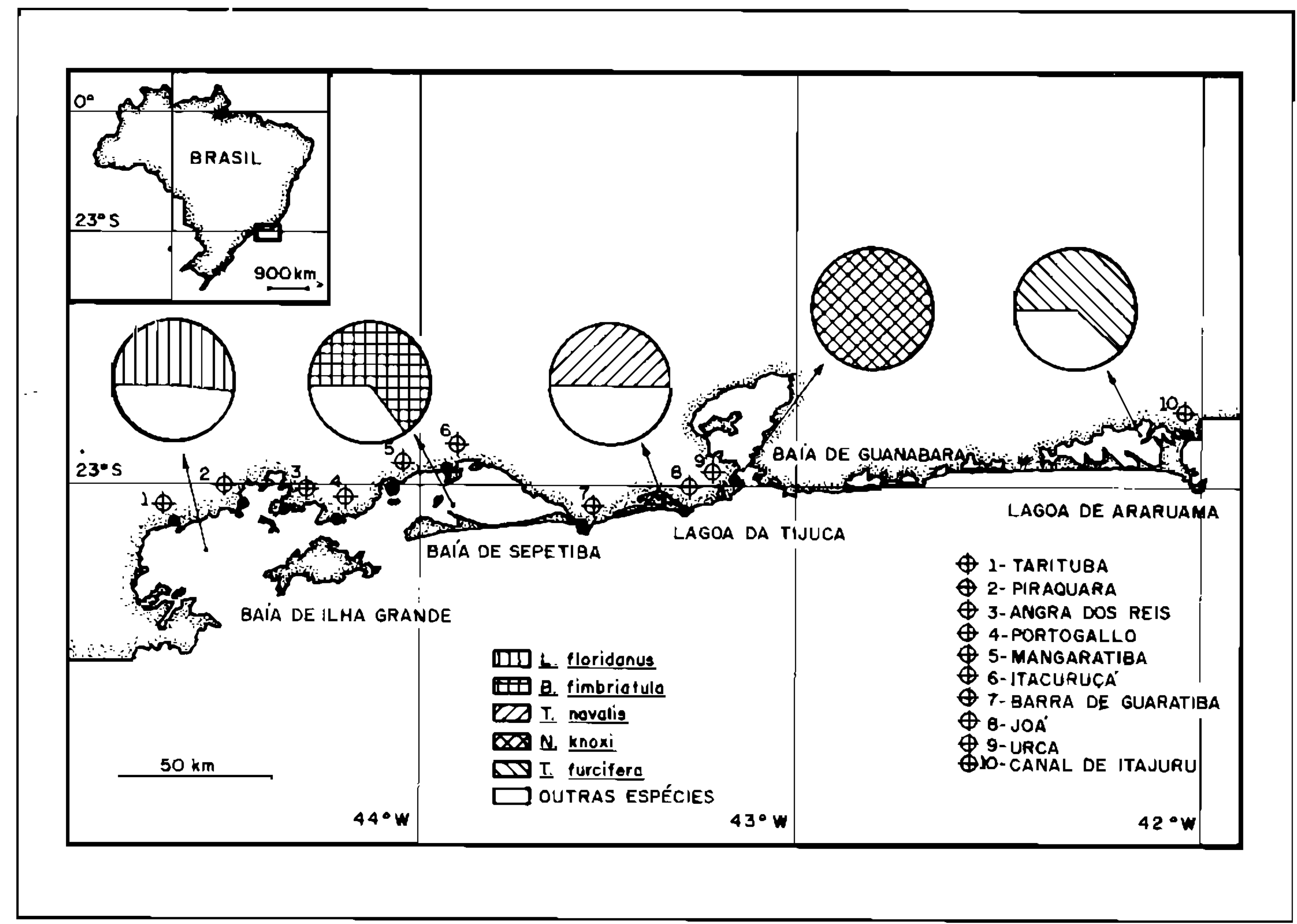

Fig. 1 : mapa da regiāo estudada demonstrando a dominância das espécies em cada área.

\section{RESULTADOS E DISCUSSÃO}

Os resultados relativos à infestação e diversidade verificados nas diversas estações de coleta encontram-se reunidos nas Tabelas I e II. Os dados obtidos para salinidade e temperatura estāo na Tabela III. Das 10 espécies de Teredinidae identificadas apenas Lyrodus affinis (Deshayes, 1863) não teve ainda sua ocorrência assinalada para a costa brasileira. As demais espécies já tiveram sua ocorrência assinalada inclusive para a costa do Rio de Janeiro (Silva, 1985; Junqueira, 1987).

A análise dos resultados demonstrou a existência de 5 áreas na costa do Rio de Janeiro que diferem entre si, tanto em relação às espécies dominantes quanto em relação ao grau de infestação. Aplicando-se o Indice de Sanders verifica-se que o dendrograma gerado (Fig. 2) indica claramente a existência dessas áreas. A primeira delas, a área da Baía de Ilha Grande formada pelas localidades de Tarituba, Piraquara, Angra dos Reis e Portogallo, onde a espécie dominante é Lyrodus floridanus (Bartsch, 1922) com uma dominância média de $51,9 \%$. A segun- da área identificada é a da Baía de Sepetiba formada pelas localidades de Mangaratiba, Itacuruçá e Barra de Guaratiba, onde a espécie mais freqüente é Bankia fimbriatula Moll \& Roch, 1931 com uma média de $64,8 \%$. A terceira área, a da Lagoa da Tijuca apresenta dominância de Teredo navalis Linnaeus, $1758 \mathrm{com} 50 \%$. A quarta área, a da Baía da Guanabara, com a dominância de $100 \%$ de Nototeredo knoxi (Bartsch, 1931) é na realidade uma região caracterizada por uma baixa infestação de Teredinidae. Na quinta área, a do Canal de Itajuru, a espécie dominante é Teredo furcifera Martens, 1894 com $61,9 \%$.

Estes resultados estão condicionados por fatores abióticos e bióticos encontrados em cada área estudada. Entre os abióticos (Tabela III) a salinidade parece ser o fator com maior determinância na definição das espécies dominantes em algumas áreas. Esta influência já foi assinalada para outras partes do mundo por diversos autores como Scheltema \& Truitt, 1954; Hoestland \& Brasselet, 1968; Culliney, 1970; Saraswathy \& Nair, 1974 e Rayner, 1979. Este fator parece ter muita importância na Baía de 


\section{TABELA I}

Número de exumplares e dominância relativa das espécies de Teredinidae encontradas nas diversas locálidades estudadas

\begin{tabular}{|c|c|c|c|c|c|c|c|c|c|c|}
\hline Espécies & 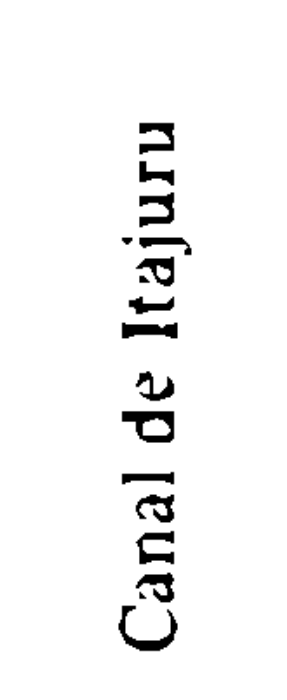 & 总 & $\stackrel{\pi}{0}$ & 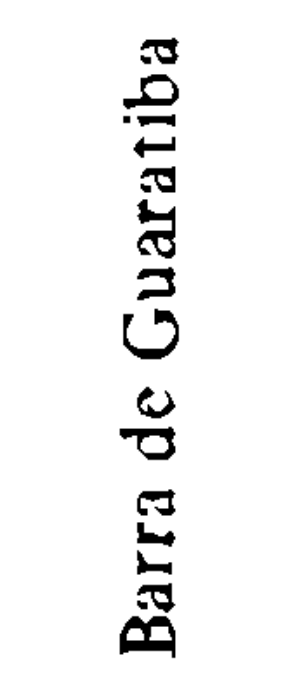 & 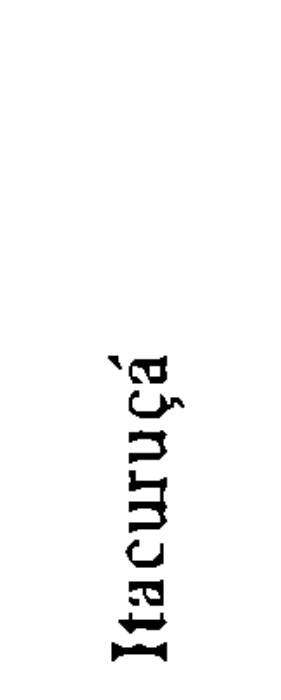 & 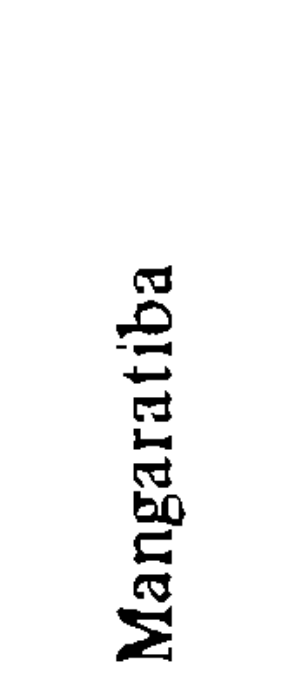 & 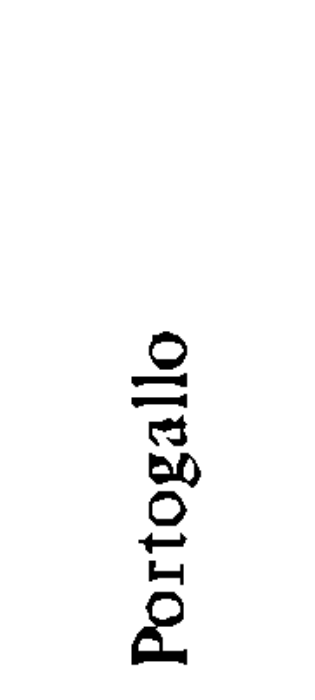 & 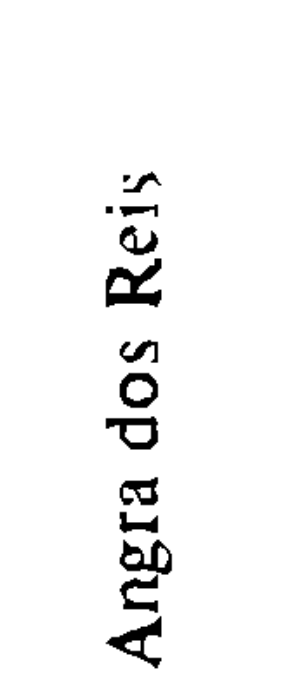 & 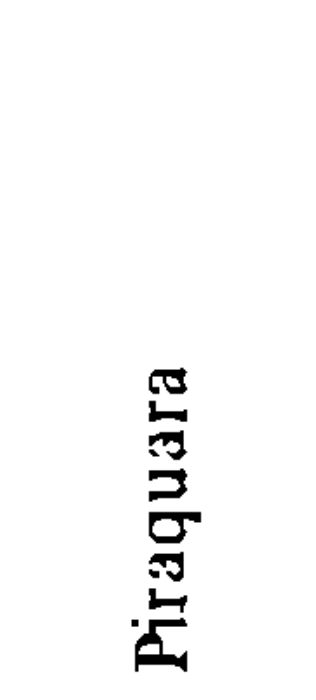 & 䄈 \\
\hline \multirow[t]{2}{*}{ Lyrodus floridanus } & 3 & - & 2 & 26 & 9 & 1 & 172 & 36 & 109 & 24 \\
\hline & $4,2 \%$ & - & $12,5 \%$ & $15 \%$ & $15 \%$ & $1,1 \%$ & $56,7 \%$ & $45 \%$ & $48,8 \%$ & $57,1 \%$ \\
\hline \multirow[t]{2}{*}{ Bankia gouldi } & 12 & - & - & 51 & 9 & 2 & 78 & 6 & 7 & - \\
\hline & $16,9 \%$ & - & - & $29.6 \%$ & $15 \%$ & $2,1 \%$ & $25.7 \%$ & $7,6 \%$ & $3,1 \%$ & .. \\
\hline \multirow[t]{2}{*}{ Teredo furcifera } & 44 & - & - & 5 & - & 1 & 19 & 20 & 24 & 2 \\
\hline & $61,9 \%$ & - & - & $2,9 \%$ & - & $1,1 \%$ & $6.3 \%$ & $25 \%$ & $10,8 \%$ & $4,8 \%$ \\
\hline \multirow[t]{2}{*}{ Teredo nal'alis } & 2 & - & 8 & 11 & 10 & - & 17 & 3 & 2 & - \\
\hline & $2,8 \%$ & - & $50 \%$ & $6,3 \%$ & $16,7 \%$ & - & $5,6 \%$ & $3,7 \%$ & $0,9 \%$ & - \\
\hline \multirow[t]{2}{*}{ Bankia fimbriatula } & 1 & - & 2 & 79 & 32 & 90 & 6 & 10 & 13 & 14 \\
\hline & $1,5 \%$ & - & $12,5 \%$ & $45,6 \%$ & $53,3 \%$ & $95,7 \%$ & $1,9 \%$ & $12,5 \%$ & $5,8 \%$ & $33,3 \%$ \\
\hline \multirow[t]{2}{*}{ Bankia carinata } & - & - & 1 & - & - & - & 6 & - & - & - \\
\hline & - & - & $6,3 \%$ & - & - & - & $1,9 \%$ & - & - & - \\
\hline \multirow[t]{2}{*}{ Nototeredo knoxi } & 1 & 2 & - & 1 & - & - & 5 & 2 & 24 & 1 \\
\hline & $1,5 \%$ & $100 \%$ & - & $0,6 \%$ & - & - & $1,6 \%$ & $2,5 \%$ & $10,8 \%$ & $2,4 \%$ \\
\hline \multirow[t]{2}{*}{ Lyrodus massa } & - & - & - & - & - & - & 1 & 1 & 44 & 1 \\
\hline & - & - & - & - & - & - & $0,3 \%$ & $1,2 \%$ & $19,8 \%$ & $2,4 \%$ \\
\hline \multirow[t]{2}{*}{ Teredo bartschi } & 5 & - & 3 & - & - & - & - & 2 & - & - \\
\hline & $7 \%$ & - & $18,7 \%$ & - & - & - & - & $2,5 \%$ & - & - \\
\hline \multirow[t]{2}{*}{ Lyrodus affinis } & 3 & - & - & - & - & - & - & - & - & - \\
\hline & $4,2 \%$ & - & - & - & - & - & - & - & - & - \\
\hline $\mathrm{N}$, identificados & 66 & - & 11 & 2 & - & 4 & 84 & 2 & 6 & 1 \\
\hline Mortos & 2 & - & - & 7 & - & 7 & 36 & 22 & 9 & 15 \\
\hline
\end{tabular}

\section{TABELA II}

Média de exemplares por coletor, densidade $\left(\mathrm{cm}^{2}\right)$, riqueza de espécies e índice de diversidade de Shannon-Wiencr ( $\left.{ }^{*}\right)$ encontrados nas 10 localidades estudadas

\begin{tabular}{|c|c|c|c|c|}
\hline Localidades & $\begin{array}{l}\text { Média de } \\
\text { excmplar } \\
\text { por coletor }\end{array}$ & $\begin{array}{l}\text { Densidade } \\
\left(\mathrm{cm}^{2}\right)\end{array}$ & $\begin{array}{l}\text { Riqueza de } \\
\text { espécies }\end{array}$ & $\mathbf{H}^{\prime}$ \\
\hline Canal de Itajuru & 27,8 & 0,69 & 8 & 0,55 \\
\hline Urca & 0.25 & 0,006 & 1 & 0 \\
\hline Juá & 5.4 & 0,13 & 5 & 0,59 \\
\hline Burra de Guaratiba & 36.4 & 0.91 & 6 & 0.57 \\
\hline Iticuruçá & 12,0 & 0,33 & 4 & 0,52 \\
\hline Mangaratiba & 21,0 & 0.52 & 4 & 0,10 \\
\hline Portogallo & 84.8 & 2.12 & 8 & 0.54 \\
\hline Angra dos Reis & 20.8 & 0,52 & 8 & 0,67 \\
\hline Piraquwa & 47,6 & 1,19 & 7 & 0,64 \\
\hline Tiurituba & 11,6 & 0,29 & 5 & 0,44 \\
\hline
\end{tabular}

Sepetiba, onde a espécie dominante é $B$. fimbriatula e onde a salinidade é normalmente baixa devido à contribuição de diversos rios de razoável porte que desaguam nesta área. Segundo os dados encontrados por vários autores (Serpa \& Karstedt, 1978; Muller, 1984) esta é uma espécie eurihalina que suporta salinidades muito baixas. Este fato foi comprovado por experimentos controlados em aquário em nosso laboratório onde a mortalidade entre estes indivíduos foi igual a zero após 4 semanas em salinidade de 7 ppm. Embora a localidade de Barra

\section{TABELA III}

Valores médios de temperatura $\left({ }^{\circ} \mathrm{C}\right)$ e salinidade (ppm) obtidos nas 10 localidades

\begin{tabular}{lcc}
\hline \multicolumn{1}{c}{ Estaçōes } & Temperatura & Salinidade \\
\hline Canal de Itajuru & 23,6 & 35,3 \\
Urca & 24,2 & 31,3 \\
Joá & 23,4 & 25,2 \\
Barra de Guaratiba & 24,5 & 32,8 \\
Itacuruçá & 25,0 & 29,8 \\
Mangaratiba & 23,5 & 26,3 \\
Portogallo & 24,0 & 34,7 \\
Angra dos Reis & 26,0 & 33,6 \\
Piraquará & 25,4 & 33,8 \\
Tarituba & 24,7 & 31,8 \\
\hline
\end{tabular}




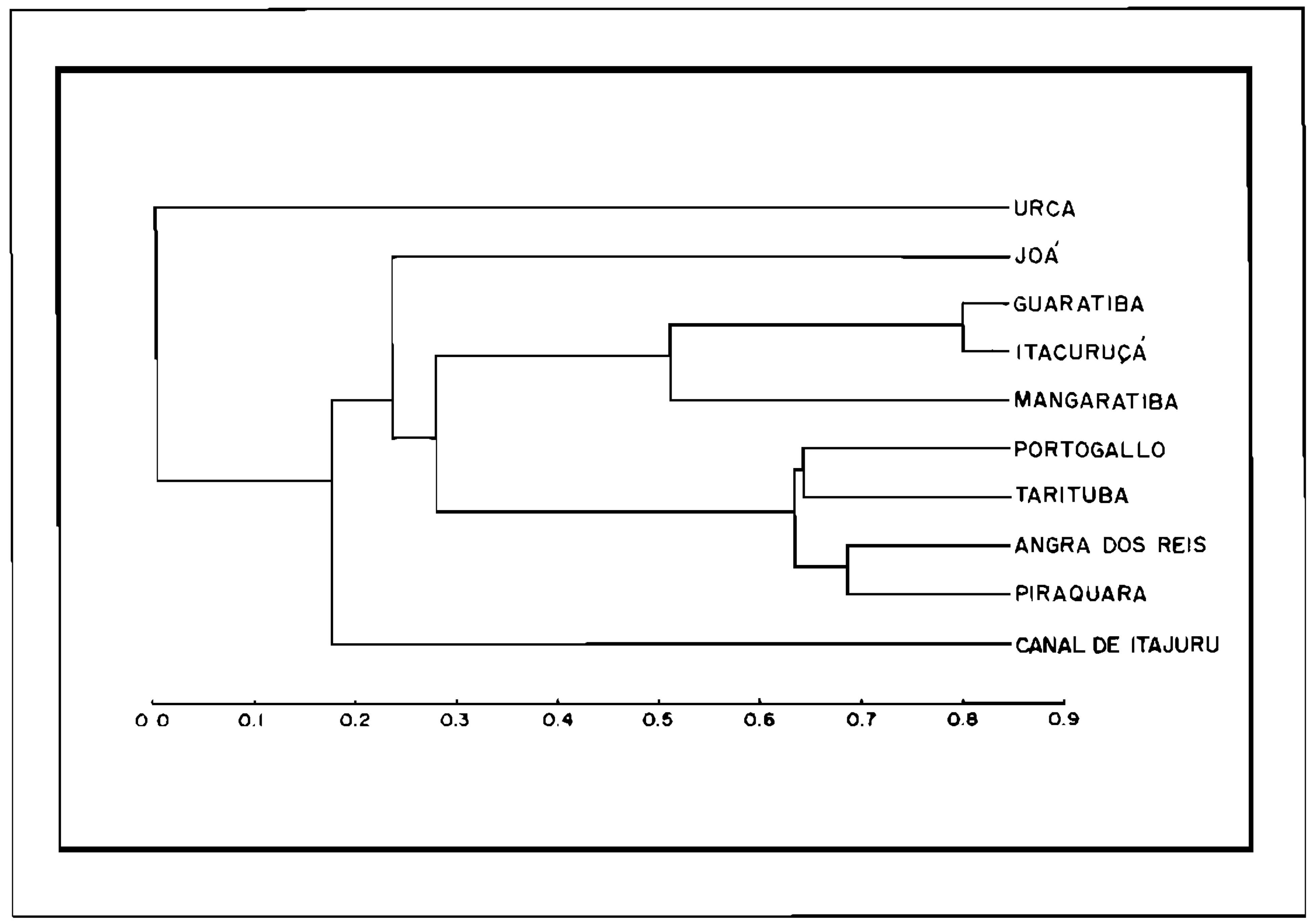

Fig. 2: dendrograma gerado através da aplicaçāo do Indice de Similaridade de Sandres entre as localidades estudadas.

de Guaratiba tenha apresentado salinidade acima de $30 \mathrm{ppm}$, isto se explica pelo fato dos coletores terem permanecido na entrada norte da baía, que se comunica com o oceano. Sendo assim, esta localidade se encontra sob influência da Baía de Sepetiba na vazante e do oceano na enchente. Sua elevada infestação está provavelmente associada à presença de extensos manguezais na área.

Outra área onde a salinidade teve um importante papel foi a Lagoa da Tijuca. Esta região estuarina apresenta grandes variações de salinidade na sua embocadura o que explicaria a dominância de $T$. navalis que apresenta alta resistência à baixas salinidades. Blum (1922) demonstrou que esta espécie tem atividade normal até salinidades de $9 \mathrm{ppm}$. Outros autores que atestaram esta resistência foram: Kofoid et al., 1927; Scheltema \& Truitt, 1954; Culliney, 1970 e 1975.

O Canal d: Itajuru é também uma região onde ocorrem grandes variaçōes de salinidade, devido ao fato de ser a comunicação da Lagoa de Araruama (que apresenta salinidades acima de
$35 \mathrm{ppm}$ ) com o mar. Apesar de experimentos realizados em aquários indicarem que a espécie dominante nesta área, $T$. furcifera, não é uma espécie resistente à baixas salinidades, esta espécie suporta as variações de salinidades mais elevadas encontradas na entrada do Canal de Itajuru.

A Baía de Guanabara é uma área onde provavelmente os fatores bióticos como a incrustação biológica têm um papel mais importante que os abióticos. Na localidade da Urca a moderada poluição orgânica aumenta a disponibilidade de nutrientes e conseqüentemente a produção primária, o que permite o desenvolvimento de grande biomassa de incrustantes filtradores. A incrustação presente nos coletores nesta área é tão intensa (Silva, 1985; Zalmon, 1988) que impede o aporte de larvas até a madeira e, quando elas ali conseguem chegar e sofrer a metamorfose, sofrem um intenso processo competitivo que virtualmente as eliminam. Além da falta de espaço disponível para penetrar na madeira, a presença de grande quantidade de filtradores principalmente ascídias (Zalmon, 1988) diminui sensivelmente a quantidade de larvas no 
plancton. Vários autores como Nagabushanan (1960) e Cooke et al. (1980) têm encontrado uma correlação negativa entre a quantidade de incrustaçāo biológica e a fixação de perfurantes. A dominância de $N$. knoxi nesta área pode ser considerada um fato inteiramente fortuito, que poderia ocorrer com qualquer outra espécie que eventualmente esteja presente nesta área como por exemplo Bankia gouldi (Bartsch, 1908), T. furcifera e Lyrodus pedicellatus (Quatrefages, 1849) de acordo com Silva et al. (1980) e Silva (1985).

$\mathrm{Na}$ Baía de Ilha Grande, a salinidade também varia pouco devido à sua ampla comunicação com o oceano. Nesta área, L. floridanus, a espécie dominante, parece ser mais estenohalina que $B$. fimbriatula e $T$. navalis como registrado por Muller (1984). Sua elevada incidência nas localidades estudadas nesta área, está associada a uma baixa incrustação biológica nos coletores e à grande disponibilidade de madeira existente na região.

Comparando-se o grau de infestação verificamos que seus valores podem ser situados desde fortemente infestados até levemente infestados de acordo com o número de exemplares por $\mathrm{cm}^{2}$ numa escala que elaboramos e que está definida na Tabela IV.

TABELA IV

Resultado da infestação das localidades estudadas na costa do Estado do Rio de Janeiro

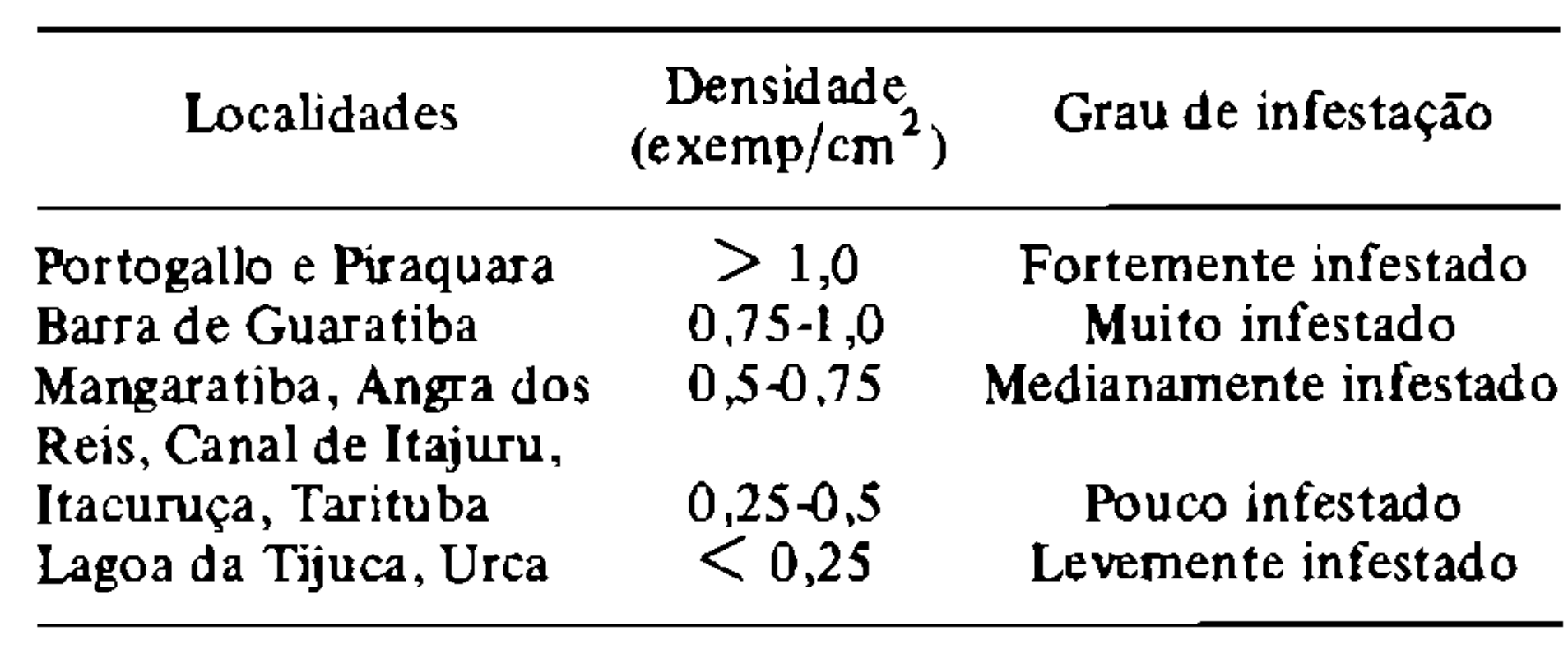

Como pode ser visualizado nesta tabela, este pode variar dentro da mesma área de dominância de uma determinada espécie. Assim numa mesma área podemos encontrar localidades desde fortemente infestadas até pouco infestadas.

De qualquer modo estes resultados demonstram que a Baía da Ilha Grande tem uma infestação superior a das demais áreas. Seguem-se a Baía de Sepetiba, Canal de Itajuru, a Lagoa da Tijuca e por fim a Urca. As diferenças dentro de uma mesma área devem-se provavelmente a fa- tores, tais como: a existência de madeira já infestada que funcionaria como foco de dispersão de larvas, a quantidade e a composição específica da comunidade incrustante e aos fatores abióticos locais.

Foram encontrados na literatura, valores de infestação situados entre 0,003 e 6,0 exemplares por $\mathrm{cm}^{2}$ incluindo áreas em regiōes temperadas e tropicais. Cabe ressaltar que são poucos os trabalhos que apresentem dados numéricos quanto à infestaçâa, principalmente no Brasil. Além da escassez de dados, a comparação é prejudicada pela diversidade dos coletores empregados em relação ao tipo e volume de madeira utilizado, diferentes metodologias de análise da infestação e tempos diferentes de imersão.

Apesar de ser citado na literatura que a atividade de perfurantes é muito mais severa em águas tropicais, esta afirmação não deve ser generalizada. Como já foi discutido, a infestaçāo é condicionada por muitos outros fatores além da temperatura. A maior diferença entre a infestação em águas tropicais e temperadas está relacionada à sazonalidade.

\section{REFERENNCIAS}

BLUM, H. F., 1922. On the effect of low salinity on. T. navalis. Univ. Calif. Publis. Zool, 22:349-368.

COOKE, W. J.; GROVHOUG, J. G. \& CHING, P. J., 1980. A survey of marine borer activity in Hawaiian nearshore waters: effects of environmental conditions and epifauna. Anales del $V$ Congresso Internacional de Corrosion Marina e incrustaciones: $155-174$.

CULLINEY, R. S., 1970. Larval biology and recnitment of the shipworms Teredo navalis and Bankia gouldi in the Newport Estuary, North Carolina. Ph. D. Thesis. Dept. Zoology, Duke Univ., N. Carolina.

CULLINEY, R. S., 1975. Comparative larval development of the shipworms Bankia gouldi and Teredo navalis. Mar. Biol, 29:345-351.

FERNANDES, L. B. M. \& COSTA, A. F., 1967. Notas sobre organismos incrustantes e perfurantes das embarcaçōes. Bol. Est. Pesca, 7: 9-26.

HOESTLAND, H. \& BRASSELET, M. P., 1968. Cycle biologique de Teredinidae saumâtres d'Afrique équatoriale occidentale Infiuence de la salinité. C. r. hebd. Seanc. Acad. Sci., Paris, 266 (D): 623685 , figs., tabs.

JUNQUEIRA, A. O. R., 1987. Estudo experimental dos Teredinidae Rafinesque, 1815 (Mollusca Bivalvia) do estuário da Lagoa da Tijuca, Rio de Janeiro. RJ. Brasil. Tese de Mestrado. Muscu Nacional. UFRJ. $251 \mathrm{pp}$.

KOFOID, C. A.; LAZIER, E. L.; DORE, W. H., BLUM, H. F.; VAN SLYKE, E. \& MILLER, R. C., 1927. Biological Section, p. 188-343. In C. L. Hill \& C. A. Koloid. Marine borers and their relation to 
marine construction on the Pacific Coast. San Francisco Bay Marine Piling Comm. San Francisco. MULLER, A. C., 1984. Organismos marinhos perfurantes de madeira do Estado do Paraná. Tese de Mestrado. Zoologia. Universidade Federal do Paraná. Curitiba. $111 \mathrm{p}$.

NAGABUSHANA, R., 1960. A note on the inhibition of marine wood boring molluscs by heavy fouling accumulation. Sci. Cult., 26: 127-128.

RAYNER, S. M., 1979. Comparation of the salinity range tolerated by teredinids (Mollusca: Teredinidae) under controlled conditions with that observed in an estuary in Papua New Guinea. Aust. J. Mar. Freshwat. Res., 30:521-533.

SANDERS, H., 1960. Benthic studies in Buzzards Bay III The structure of the soft-bottom communities. Limnol. Oceanogr., 5: 138-153.

SARASWATHY, W. \& BALAKRISHNAN, N., 1974. The influence of salinity on a tropical estuarine shipworm Nausitora hedleyi Schepman (Bivalvia: Teredinidae). Hydrobiologia, 44: 397-411.

SCHETELMA, R. S. \& TRUITT, R. V., 1954. Ecological factors related to the distribution of Bankia gouldi Bartsch in Chesapeake Bay. Chesapeake Biol. Lab. Publ., 100: 1-31.

SERPA, F. G. \& KARSTEDT, P., 1978. Natural resistance to marine borers of nine North and Northeastern Brazilian wood species. Floresta, Curitiba,
9:97-102

SILVA, S. H. G., 1985. Estudo experimental sobre a infestação de perfurantes de madeira na costa do Estado do Rio de Janeiro. Tese de Doutorado. Fisiologia Animal. Instituto de Biociências. Universidade de São Paulo. São Paulo. 231 p.

SILVA, S. H. G.; NUNES, A. J. B.; ALVES, M. C. S \& LAGE, V. A., 1980. Contríbuição ao estudo das comunidades incrustantes que ocorrem na Baía da Guanabara, Rio de Janeiro, Brasil. Resultados preliminares. Rev. Brasil. Biol., 40: 367-382.

TURNER, R. D., 1971. Identification of marine wood boring molluses, p. 17-84. In E. B. G. Jones \& S. K. Eltringhan, Marine borers, fungi and fouling organisms of wood. Org. Econom. Coop. Develop. Paris.

TURNER, R. D. \& JOHNSON, A. C., 1971. Biology of marine wood boring molluscs, p. 259-301, figs., tabls. In E. B. G. Jones \& S. K. Eltringham, Marine borers, fungi and fouling organisms of wood. Org. Econom. Coop. Develop. Paris.

WASHINGTON, H. G., 1984. Diversity, biotic and similarity indices. A review with special relevance to aquatic ecosystems. Waters Res., 18: 653-693.

ZALMON, I. R., 1988. Estudo de comunidades incrustantes sobre painéis experimentais em três áreas da Baía de Guanabara, RJ, Brasil. Tese de mestrado. Zoologia. Museu Nacional. 195 p. 